\title{
Dynamics of local adaptation in a stable environmental gradient
}

\author{
Edvin Memet \\ Department of Physics, Harvard University, Cambridge, Massachusetts 02138, USA
}

Ned S. Wingreen $\odot$

Lewis-Sigler Institute for Integrative Genomics, Princeton University, Princeton, New Jersey 08544, USA and Department of Molecular Biology, Princeton University, Princeton, New Jersey 08544, USA

\author{
Yigal Meir \\ Department of Physics, Ben-Gurion University of the Negev, Beer Sheva 84105, Israel \\ and Department of Physics, Princeton University, Princeton, New Jersey 08544, USA
}

(Received 28 March 2021; revised 13 September 2021; accepted 12 October 2021; published 17 November 2021)

\begin{abstract}
Microbial populations in nature generally inhabit extended environments with substantial spatial variation in ecological factors: light intensity in the ocean, temperature in geothermal hot springs, or a variety of chemical concentrations including salt and $p \mathrm{H}$. In such continuously varying environments, it remains unclear why a finite number of subpopulations form and how this number is set. Here we show that a model of asexual evolution in a gradient maps onto a no-gradient neutral model, and by mapping this model to a gas of kinks and antikinks, we derive the full distribution of the number of coexisting lineages, and their correlation functions. Testing these predictions in controlled laboratory experiments would provide valuable insights into many real-world microbial communities.
\end{abstract}

DOI: 10.1103/PhysRevResearch.3.L042026

A main tenet of evolution is that each species adapts to local conditions in order to maximize its chances of survival and growth. Even a single bacterial species may evolve into different ecologically distinct lineages [1-4] depending on environmental conditions. For instance, in the "evolution canyons" of Israel multiple lineages that would conventionally be classified as a single species have been confirmed to be ecologically distinct, specializing to distinct canyon slopes receiving different levels of solar exposure $[5,6]$. Similarly-though in a continuous environmentSynechococcus subclades in hot spring cyanobacterial mats [Fig. 1(a)] were found to be uniquely adapted to specific temperatures $[3,7]$. Other spatially graded environments include, for example, light intensity in the ocean [8] or estuarine salinity gradients [9].

The latter examples raise an interesting question: if each subclade occupies a distinct temperature interval, competitively excluding all other subpopulations, what will happen in graded environments - which provide a continuum of ecological niches? Do we expect to see continuous adaptation, or will we observe a finite number of distinct lineages? In the latter case, what will then determine the typical extent of a lineage as well as the distribution of lineage sizes? Here, starting from

Published by the American Physical Society under the terms of the Creative Commons Attribution 4.0 International license. Further distribution of this work must maintain attribution to the author $(s)$ and the published article's title, journal citation, and DOI. a model that incorporates real-space diffusion, mutations, and selection in a linearly spatially varying time-stable environment, we show, both analytically and numerically, that such a model maps onto a simpler model in a neutral environment. By mapping the neutral model onto a gas of kinks and antikinks, we calculate exactly the distribution of lineage sizes and their pair correlation function, which agree with numerical results from the full model. Our results can prove useful for future studies of evolution in both natural environments and in experimentally produced spatial gradients.

Gradient model. We consider a one-dimensional (1D) population, confined to a region of size $L$. All lengths will be scaled by $L$, so the position of each individual is $x \in[0,1]$. We assume that a single ecological factor, which we term "temperature," dominates, exhibiting the strongest gradient, $T(x)=\alpha x$. Individuals are characterized by a temperature phenotype $\phi_{T}$, which corresponds to their preferred growth temperature [Fig. 1(b)], such that an individual at position $x$ with temperature phenotype $\phi_{T}$ has fitness $w\left(x, \phi_{T}\right)=$ $\exp \left[-\left(x-\phi_{T} / \alpha\right)^{2} /\left(2 \sigma_{s}^{2}\right)\right]$ and growth rate $w\left(x, \phi_{T}\right) \ln 2 / \tau_{g}$, where $\tau_{g}$ is the generation time (see Sec. S1 in Ref. [10] for a list and description of parameters used in this Letter). Migration is characterized by a spatial diffusion coefficient $D_{x}=\sigma_{x}^{2} / \tau_{g}$, where $\sigma_{x}$ is the typical displacement due to migration in one generation's time.

We initially examine a model with only locally adaptive mutations which we denote as the "gradient model." Local mutations stochastically change the optimal phenotype $\phi_{T}$ upon some cell divisions, without changing the maximal fitness. These mutations are characterized by a phenotype 

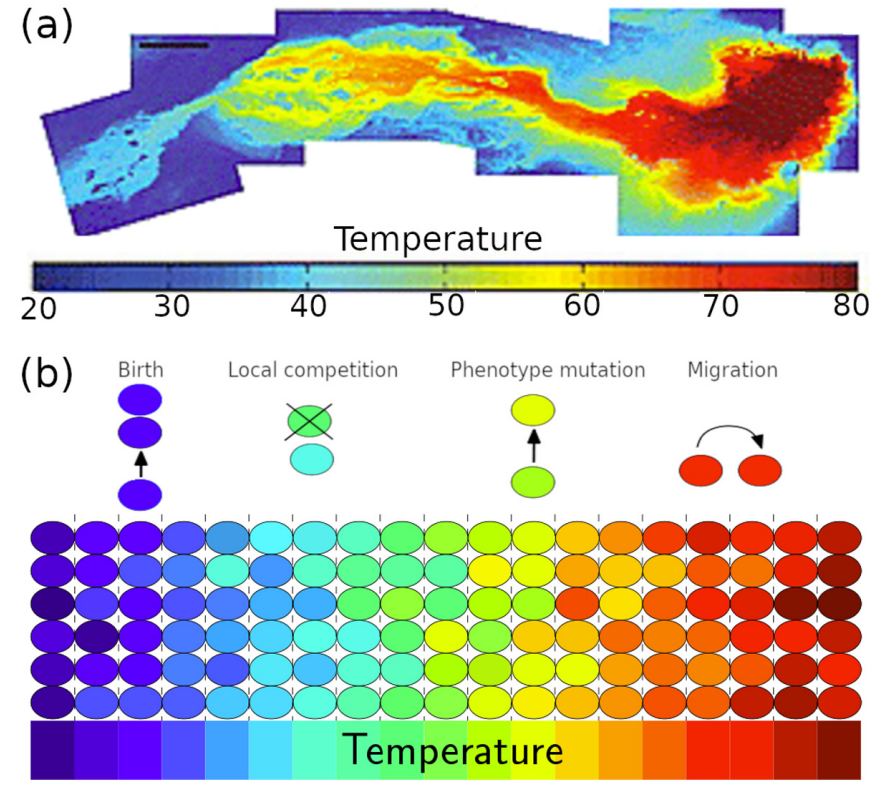

FIG. 1. (a) Temperature gradient in a hot spring within the El Tatio Geyser Field, Chile, revealed through the use of thermal infrared imaging. The black scale bar represents 0.5 meters [11]. (b) Schematic of model for cells evolving in temperature gradient. The color of each cell represents its temperature phenotype (i.e., preferred growth temperature) while the color bar shows environmental temperature (and spatial location). The better the color match between a given cell's preferred growth temperature and the local environmental temperature, the higher the fitness of that cell. Top: the allowed processes within the model

diffusion rate $D_{\phi}$. Mutation and migration, together with selection, induce phenotypic and spatial variances of the population around the optimal line $\phi_{T}=\alpha x$. We refer to the spatial scale of this distribution around the optimum as the "phenotype width" $\sigma_{p}$.

In the continuum limit of our model, the change in the density $c\left(x, \phi_{T}\right)$ of phenotype $\phi_{T}$ located at $x$ satisfies

$$
\begin{aligned}
\frac{\partial c\left(x, \phi_{T}\right)}{\partial t}= & \frac{c\left(x, \phi_{T}\right)}{\tau_{g} / \ln 2}\left[w\left(x, \phi_{T}\right)-\int d \phi_{T}^{\prime} w\left(x, \phi_{T}^{\prime}\right) c\left(x, \phi_{T}^{\prime}\right)\right] \\
& +D_{x} \frac{\partial^{2} c\left(x, \phi_{T}\right)}{\partial x^{2}}+D_{\phi} \frac{\partial^{2} c\left(x, \phi_{T}\right)}{\partial \phi_{T}^{2}}
\end{aligned}
$$

where the terms on the right-hand side represent, respectively, net growth (composed of the growth term $w\left(x, \phi_{T}\right)$ and a death term which keeps the total density fixed at a given location $x$ ), migration, and mutation. Without loss of generality, we normalize the density such that $\int d \phi_{T} c\left(x, \phi_{T}\right)=1$.

Mapping to no-gradient model. At steady state, the relative difference between the growth rate of a specific phenotype and the average growth rate of all phenotypes in that location [the first term on the right in Eq. (1)] is compensated by diffusion in both real and phenotype spaces (the additional terms). Consider a phenotype at its optimal location, such that $w=1$. Given that the population of any phenotype is confined to a typical distance of $\sigma_{p}$ around its optimal position, the average fitness of all phenotypes at any specific location is typically $\exp \left(-\beta \sigma_{p}^{2} / \sigma_{s}^{2}\right)$, with $\beta$ some

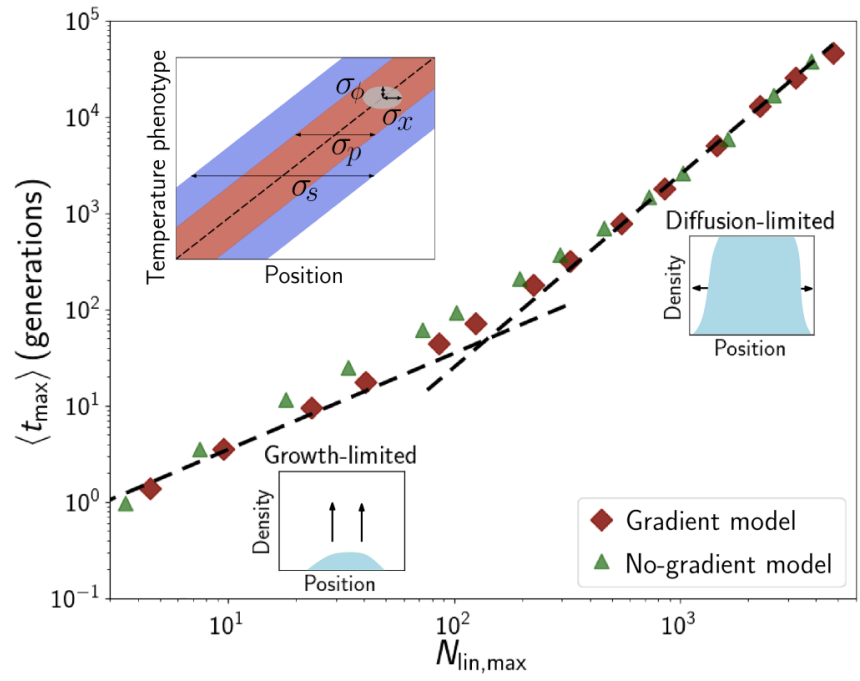

FIG. 2. Model with locally adaptive mutations: The average time from simulation at which a lineage reaches its maximum size as a function of that maximum size, for the gradient model with local mutations (red diamonds), and the rescaled no-gradient model (green triangles). Dashed lines of slope 1 and 2 serve as indicators of linear-in-time and diffusive growth regimes, respectively. $\mathrm{Pa}$ rameters: $D_{x}=2.1 \times 10^{-5}, D_{\phi} / \alpha^{2}=2.3 \times 10^{-6}, \sigma_{s}=0.01$ for the gradient model; $D=2.3 \times 10^{-6}$ for the no-gradient model; population size $N=50000$ and deme size $n=10$, throughout. Top inset: Illustration of the mapping from evolution with locally adaptive mutations in a gradient to one-dimensional neutral evolution (not to scale): since the resulting phenotype width $\sigma_{p}$ (red strip) is much smaller than the selection length $\sigma_{s}$ (black strip), at steady state all cells are approximately equally fit, meaning the population is effectively neutral. The gray ellipse represents the typical spreading in one generation's time $\tau_{g}$ due to migration and mutations. Bottom insets: Schematics of typical spatial profiles of lineages in the linear (left) and diffusive (right) growth regimes.

numerical constant. For phenotype width $\sigma_{p}$, the diffusion terms are typically equal to $\approx\left(D_{x}+D_{\phi} / \alpha^{2}\right) / \sigma_{p}^{2}$, so that in steady state $1-\exp \left(-\beta \sigma_{p}^{2} / \sigma_{s}^{2}\right) \approx D_{\Sigma} \tau_{g} /\left(\ln 2 \sigma_{p}^{2}\right)$, with $D_{\Sigma}=$ $D_{x}+D_{\phi} / \alpha^{2}$. Since we expect that the typical distance covered in one generation, either in real space or in phenotype space, will be much smaller than $\sigma_{s}$ (otherwise all progeny would immediately die), we find $\sigma_{p} \sim \sqrt{\sigma_{s} \sqrt{D_{\Sigma} \tau_{g}}}$, where all numerical constants have been dropped. In the reasonable limit where diffusion in phenotype space (scaled by $\alpha$ ) is much slower than diffusion in real space, this equation reduces to the result found by Felsenstein [12] for the typical phenotype width in the absence of any environmental gradient: $\sigma_{p} \sim \sqrt{\sigma_{s} \sigma_{x}}$. A more rigorous derivation of this result is provided in Sec. S2 in Ref. [10], where we also confirm this scaling by simulations (Fig. S1).

The resulting separation of length scales, $\sqrt{D_{\Sigma} \tau_{g}} \ll \sigma_{p} \ll$ $\sigma_{s}$ is illustrated in Fig. 2 (inset). Notably, if selection is strong on intermediate timescales, the population is effectively confined to a ridge along the diagonal that is narrow relative to the selection scale. Accordingly, all cells are approximately equally fit such that the population sees an effectively neutral environment-i.e., in this parameter regime, the gradient 
model maps to an effective no-gradient model. Individuals that venture outside the region of neutrality are eventually removed from the population by selection, in a process akin to the purifying/background selection phenomenon $[13,14]$. The narrow phenotype width $\sigma_{p}$ also implies that meaningful spreading takes place only along the optimal adaptation line $\phi_{T}=\alpha x$ (black dashed line) and the effective rate $D$ satisfies $1 / D=1 / D_{x}+\alpha^{2} / D_{\phi}$. Thus, for $D_{\phi} / \alpha^{2} \ll D_{x}$, we get $D \approx$ $D_{\phi} / \alpha^{2}$, i.e., the effective diffusion rate is set by the slower diffusion mode.

As a strong test of this correspondence, we have simulated our model and compared, in Fig. 2, the average time $\left\langle t_{\max }\right\rangle$ at which a lineage reaches its maximum size $N_{\text {lin,max }}$, as a function of that maximum size, for the full model with a temperature gradient (red diamonds), and the rescaled no-gradient model with uniform environment and effective spatial diffusion $D=D_{\phi} / \alpha^{2}$ (green triangles). In our simulations, space is discretized into a finite number of demes, typically with deme size of ten cells. (Results are not sensitive to deme size as long as demes are sufficiently large to render genetic drift negligible at the scale of the typical fitness differential within a deme-see Sec. S7 in Ref. [10].) For the no-gradient model one expects that growth will initially be linear in time (black dashed line of slope 1) until a critical size is reached (see Sec. S3 in Ref. [10]). This point marks a crossover to diffusive growth (black dashed line of slope 2), wherein net lineage growth can only occur at the boundaries of the domain, which spreads diffusively (insets). Indeed the rescaled model and the full model both exhibit this behavior and agree with each other, which supports the validity of the mapping.

Gradient model $(+G)$. We have seen that the model with locally adaptive mutations reaches a steady state in which each phenotype has a small spatial spread around its optimal location along the gradient. Thus, in this model, all possible phenotypes are allowed to coexist. However, some mutations may improve fitness more generally $[15,16]$. We therefore allow for the occurrence (at rate $\mu$ ) of globally adaptive mutations which do not change a cell's optimal temperature but rather increase its growth rate by a factor $1+s$. Since the individuals with globally adaptive mutations have higher maximal growth rate than the background population, they are capable, once established, of expanding over a wider spatial range, outcompeting neighboring phenotypes, and spreading. Consequently, global fitness mutations broaden the spatial extent of some lineages while bringing about the extinction of the remaining ones.

Is the mapping of the full model with a temperature gradient to a no-gradient model with rescaled diffusion still valid in the presence of globally beneficial mutations? In that case, the no-gradient model would comprise a population diffusing in a spatially uniform 1D environment, receiving globally beneficial mutations at some rate $\mu$. The spread of a given advantageous mutation in a neutral environment was studied by Fisher and Kolmogorov [17-21] who showed that, after a transient period, the boundary of the more fit domain reaches a stationary shape and constant speed $v_{x} \sim \sqrt{D_{x} s / \tau_{g}}$. To answer the question, we first consider the case when mutations are rare, so a single global mutation has time to sweep the entire environment. In this case for the no-gradient $(+\mathrm{G})$ model, we can make a simple prediction: since the two (left and right) expanding fitness fronts propagate at constant speed, the rate of lineage growth is also constant until it is suddenly halved when one of the fronts reaches the boundary of the environment, and finally drops to zero when the second front hits the other boundary. Taking the average over all possible initial lineage locations, we obtain

$$
\frac{\left\langle N_{\text {lin }}(t)\right\rangle}{N}=\frac{1}{N} \frac{\int N_{\text {lin }}(t \mid x) d x}{L}=2 \hat{t}-\hat{t}^{2},
$$

where nondimensionalized time $\hat{t}=t /\left(L / v_{x}\right)$, and the lineage size conditional on initial lineage location $N_{\text {lin }}(t \mid x)$ is a piecewise function that depends on when the left- and right-traveling fronts reach the boundaries. This prediction [Fig. 3(a), black dashed curve] agrees well with the curve obtained from simulating the gradient model $(+\mathrm{G})$ (red diamonds), confirming that the mapping to a no-gradient model with no phenotype variable is still valid after introducing global mutations.

In the general case, new globally beneficial mutations can occur while previous ones have not yet taken over the environment, resulting in dynamics of lineage domains which nucleate, spread, and collide [Fig. 3(b)]. Such a no-gradient model has been examined in a different biological context by Ralph and Coop [20], who employed a crystal growth mapping in their analysis. Here we use a different mapping, which will lead to equivalent results but will also enable us to derive additional results regarding the distribution of lineage sizes. Our mapping begins with the observation that the boundaries of lineage domains move with constant speed, and are thus equivalent to a 1D gas of particles or "kinks" and antiparticles or "antikinks" which nucleate and annihilate in pairs [22,23]. The dynamics of this gas can be mapped onto a forced and overdamped sine-Gordon soliton gas [23] allowing us to obtain $P\left(\ell_{n}\right)$, the full distribution of the spatial extent of lineage domains, where $\ell_{n}$ denotes the size of a given domain. As we show below, this distribution agrees with the distribution obtained from numerical simulations of the full model.

To obtain the expected distribution of domain sizes, we note that at any given time, all positions of kinks and antikinks are totally random [23]. Thus finding the distribution of niche sizes $P\left(\ell_{n}\right)$ reduces to finding the distribution of spacings between uniformly distributed random points on a line, which is a well known mathematical problem (for a review, see, e.g., [24]). The results are

$$
\begin{aligned}
P\left(\ell_{n}\right) & =N_{n}\left(1-\ell_{n}\right)^{N_{n}-1}, \\
P_{2}\left(\ell_{n 1}, \ell_{n 2}\right) & =N_{n}\left(N_{n}-1\right)\left(1-\ell_{n 1}-\ell_{n 2}\right)^{N_{n}-2},
\end{aligned}
$$

where $N_{n}=L /\left\langle\ell_{n}\right\rangle$ is the average number of lineages, and we also quote the probability $P_{2}\left(\ell_{n 1}, \ell_{n 2}\right)$ of having simultaneously two lineage domains of sizes $\ell_{n 1}$ and $\ell_{n 2}$ (all higher correlation functions can also be calculated). The functional forms for both lineage size $P\left(\ell_{n}\right)$ [Eq. (3a)] and pair correlation $P_{2}\left(\ell_{n 1}, \ell_{n 2}\right)$ [Eq. (3b)] fit well the respective distributions estimated from simulation [Figs. 4(a) and 4(b), respectively], with a single fitting parameter $N_{n}$. Thus, the mapping of the full model, including growth and diffusion in an environmental gradient, with both phenotype and globally beneficial mutations, onto a model in a spatially uniform 

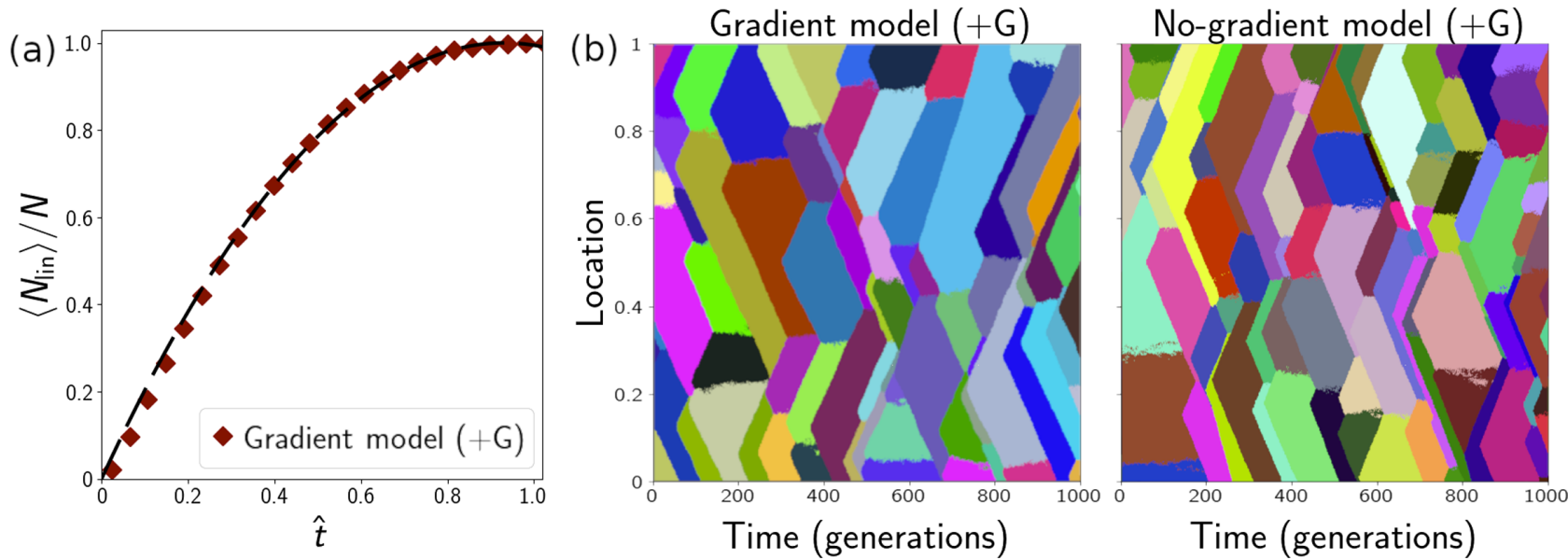

FIG. 3. (a) Comparison of the average growth dynamics of a more-fit lineage versus time, $\hat{t}=t /\left(L / v_{x}\right)$, for the gradient model $(+\mathrm{G})$ (red diamonds) versus the prediction for the rescaled no-gradient model $(+\mathrm{G})$ [Eq. (2), dashed black curve]. Parameters: $N=5000, s=0.1$, $D_{x}=2 \times 10^{-5}, D_{\phi}=2 \times 10^{-6}$. (b) Space-time evolution of interfering domains of globally beneficial mutations in the gradient model (left) and the no-gradient model (right). Each bin that is occupied in absolute majority by a single lineage is assigned a random color associated with that lineage. Parameters: $N=100000, s=0.25, \mu=3 \times 10^{-7}$ throughout; gradient model $(+\mathrm{G}) D_{x}=1 \times 10^{-6}, D_{\phi} / \alpha^{2}=1 \times 10^{-7}$; no-gradient model $(+\mathrm{G}) \mathrm{D}=1 \times 10^{-7}$.

environment, and the description of the boundaries of the lineage domains in terms of kinks and antikinks arising in a forced and overdamped sine-Gordon soliton gas, allowed us to derive analytical expressions for the exact distribution and all the correlation functions of lineage sizes in such an environment. (Note that in the kink-antikink gas, a kink and an antikink will annihilate each other when they collide, which in our model corresponds to two lineages with the same number of advantageous mutations merging when their boundaries meet, rather than coexisting as distinct lineages. The agreement between the distribution obtained from simulation and the soliton gas prediction indicates that such processes are sufficiently rare.)

$N_{n}$ can be found a priori by equating the nucleation rate $\mu N s$ (where the factor of $s$ accounts for the establishment probability, i.e., the probability that a beneficial mutation will escape genetic drift) and the annihilation rate. Since the typical time a given domain travels before it collides with another
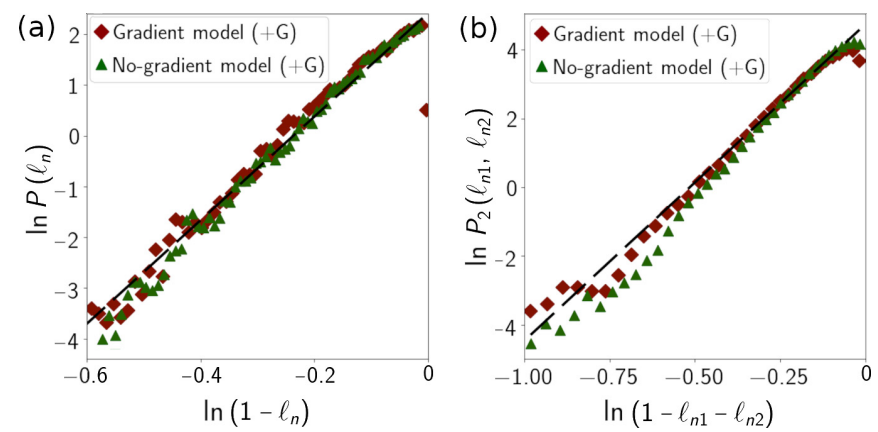

FIG. 4. Comparison of the distribution (a) and correlation function (b) of the gradient model $(+\mathrm{G})$ that includes both local and global mutations (red diamonds) and the corresponding no-gradient model (green triangles) to the predicted behavior from a forced, damped soliton gas [Eq. (3)] with $N_{n}=11.2$ (black dashed line). Parameters are identical to those in Fig. 3(b). one is $L /\left(N_{n} v_{x}\right)$, the total annihilation rate scales as $N_{n}^{2} v_{x} / L$. Therefore

$$
N_{n} \sim \sqrt{\mu N L \alpha \sqrt{\frac{s / \tau_{g}}{D_{\phi}}}}
$$

Notably, the number of lineages $N_{n}$ scales with the square root of the gradient steepness $\alpha$, scales weakly with the fitness effect $s$ of global mutations, and is independent of the rate of spatial diffusion $D_{x}$. Since the number of lineages domains cannot be smaller than 1, Eq. (4) implies a critical gradient slope $\alpha_{\mathrm{cr}} \sim\left(D_{\phi} \tau_{g} / s\right)^{1 / 2} /(\mu N L)$ such that above this value, the average domain size $\left\langle\ell_{n}\right\rangle$ is generally finite, independent of system size, and scales inversely with the square root of population density $N / L$. Below this critical slope, a single lineage can spread through the whole system and $\left\langle\ell_{n}\right\rangle$ approaches the system size $L$.

Naturally, one may ask whether it is the interference regime or the rare mutation regime that is more relevant in practice. Under no-gradient conditions, there are many instances in which clonal interference has been shown to be relevant [25] - most famously in Lenski's well-mixed experiments [26], but also in spatially structured populations [19,27]. Quantitatively, the condition for clonal interference under nogradient conditions can be written as $\mu N L \sqrt{\frac{s / \tau}{D_{x}}} \gtrsim 1$. In our model, the equivalent condition reads $\mu^{\prime} N L \sqrt{\frac{s / \tau}{D_{\phi} / \alpha^{2}}} \gtrsim 1$, with $D_{x}$ replaced by $D_{\phi} / \alpha^{2}$ and the mutation rate $\mu$ replaced by the rate of globally adaptive mutations $\mu^{\prime}$. There is evidence suggesting that the supply of hill-climbing mutations is abundant, such that microbial populations continue to rapidly adapt over long timescales even in simple constant environments without typically reaching a fitness peak $[25,28]$. This further indicates that the regime of multiple interfering lineages is likely to be widespread.

Thus, the kink-antikink gas analogy allows us to make additional statements concerning the time development of 
the population: (1) The rate at which the front expands in phenotype (preferred growth temperature) space, i.e., the "adaptation rate," is $v_{\phi}=\alpha v_{x} \sim \sqrt{D_{\phi} s / \tau_{g}}$. Interestingly, this rate of adaptation is independent of the gradient steepness $\alpha$, in agreement with the result obtained by Hermsen in the infinite population, steep gradient limit [29]. (2) The average rate of fitness increase in the population $\langle\dot{F}\rangle$ depends on density, not on population size per se: $\langle\dot{F}\rangle \sim$ $\mu N s^{2}\left\langle\ell_{n}\right\rangle \sim\left[\mu^{2}(N / L)^{2} s^{7} D_{\phi} / \alpha^{2}\right]^{1 / 4}$. This generalizes to the case of gradient environments the result obtained by Martens and Hallatschek [19] for the rate of fitness increase in spatially uniform conditions.

(3) While the distribution of lineage sizes is constant in time, the precise identity of surviving lineages continuously turns over. The turnover timescale, defined as the time it takes for average fitness to increase by $s$, is $T_{\text {turnover }}=s /\langle\dot{F}\rangle$, which is, again, independent of population size. Understanding the timescale of lineages turnover is of practical importance as it determines the degree to which distributions of microbial taxa reflect the influence of past events as opposed to contemporary environmental conditions [30].

To summarize, in this paper we have established a simple correspondence between evolution in a linear environmental gradient and evolution in a spatially uniform temporally stable environment. We used this framework to not only match a number of existing results but also to efficiently derive results, proving the usefulness of the framework for studying evolution in a gradient. In particular, we hope our results can be useful for laboratory studies, as controlled spatial gradients can be produced in vitro using, for instance, microfluidic devices that establish temperature or chemical gradients [31,32], or benthic gradient chambers capable of mimicking the oxygen, sulfide, and light gradients present in microbial mats $[33,34]$. Studying evolution in spatial gradients under simplified, carefully controlled laboratory settings may prove essential to understanding - and ultimately predicting - the emergence of drug resistance in bacteria experiencing antibiotic concentration gradients $[29,35,36]$, or the patterns of biodiversity in microbial communities [37-41].

Acknowledgments. This work was supported in part by the National Science Foundation under Grants No. PHYS1607612, No. PHYS-1066293, and No. 1853602, and through the Center for the Physics of Biological Function (Grant No. PHY-1734030) and performed in part at the Aspen Center for Physics, supported by National Science Foundation Grant No. PHY-1607611.
[1] M. Achtman and M. Wagner, Microbial diversity and the genetic nature of microbial species, Nat. Rev. Microbiol. 6, 431 (2008).

[2] D. Gevers, F. M. Cohan, J. G. Lawrence, B. G. Spratt, T. Coenye, E. J. Feil, E. Stackebrandt, Y. V. de Peer, P. Vandamme, F. L. Thompson, and J. Swings, Re-evaluating prokaryotic species, Nat. Rev. Microbiol. 3, 733 (2005).

[3] D. M. Ward, F. M. Cohan, D. Bhaya, J. F. Heidelberg, M. Kuhl, and A. Grossman, Genomics, environmental genomics and the issue of microbial species, Heredity 100, 207 (2007).

[4] F. M. Cohan, Towards a conceptual and operational union of bacterial systematics, ecology, and evolution, Philos. Trans. R. Soc. B 361, 1985 (2006).

[5] N. Connor, J. Sikorski, A. P. Rooney, S. Kopac, A. F. Koeppel, A. Burger, S. G. Cole, E. B. Perry, D. Krizanc, N. C. Field, M. Slaton, and F. M. Cohan, Ecology of speciation in the genus bacillus, Appl. Environ. Microbiol. 76, 1349 (2010).

[6] A. Koeppel, E. B. Perry, J. Sikorski, D. Krizanc, A. Warner, D. M. Ward, A. P. Rooney, E. Brambilla, N. Connor, R. M. Ratcliff, E. Nevo, and F. M. Cohan, Identifying the fundamental units of bacterial diversity: A paradigm shift to incorporate ecology into bacterial systematics, Proc. Natl. Acad. Sci. USA 105, 2504 (2008).

[7] F. M. Cohan and D. M. Ward, Microbial diversity in hot spring cyanobacterial mats: pattern and prediction, in Microbial Diversity in Hot Spring Cyanobacterial Mats: Pattern and Prediction, Geothermal biology and geochemistry in Yellowstone National Park, edited by W. P. Inskeep and T. R. McDermott (Montana State University Publications, Bozeman, MT, 2005), pp. 185201.

[8] Z. I. Johnson, E. R. Zinser, A. Coe, N. P. McNulty, E. M. S. Woodward, and S. W. Chisholm, Niche partitioning among prochlorococcus ecotypes along ocean-scale environmental gradients, Science 311, 1737 (2006).

[9] B. C. Crump, C. S. Hopkinson, M. L. Sogin, and J. E. Hobbie, Microbial biogeography along an estuarine salinity gradient: combined influences of bacterial growth and residence time, Appl. Environ. Microbiol. 70, 1494 (2004).

[10] See Supplemental Material at http://link.aps.org/supplemental/ 10.1103/PhysRevResearch.3.L042026 for parameters and additional details on the calculations.

[11] A. E. Dunckel, M. B. Cardenas, A. H. Sawyer, and P. C. Bennett, High-resolution in-situ thermal imaging of microbial mats at El Tatio Geyser, Chile shows coupling between community color and temperature, Geophys. Res. Lett. 36, L23403 (2009).

[12] J. Felsenstein, Multivariate normal genetic models with a finite number of loci, Multivariate Normal Genetic Models with a Finite Number of Loci, Technical Report No. RLO-2225/5-39, Department of Genetics, Washington University, Seattle, 1977.

[13] C. B. Charlesworth, Deborah and M. T. Morgan, The effects of genetic and geographic structure on neutral variation, Genetics 141, 1619 (1995).

[14] I. Cvijović, B. H. Good, and M. M. Desai, The effect of strong purifying selection on genetic diversity, Genetics 209, 1235 (2018).

[15] M. M. Desai and D. S. Fisher, Beneficial mutation selection balance and the effect of linkage on positive selection, Genetics 176, 1759 (2004).

[16] B. H. Good, S. Martis, and O. Hallatschek, Adaptation limits ecological diversification and promotes ecological tinkering during the competition for substitutable resources, Proc. Natl. Acad. Sci. USA 115, E10407 (2018). 
[17] R. A. Fisher, The wave of advance of advantageous genes, Ann. Hum. Genet. 7, 355 (1937).

[18] A. Kolmogorov, I. Petrovskii, and N. Piscunov, A study of the equation of diffusion with increase in the quantity of matter, and its application to a biological problem, Byul. Moskovskogo Gos. Univ. 1, 1 (1937).

[19] E. A. Martens and O. Hallatschek, Interfering waves of adaptation promote spatial mixing, Genetics 189, 1045 (2011).

[20] P. Ralph and G. Coop, Parallel adaptation: one or many waves of advance of an advantageous allele? Genetics 186, 647 (2010).

[21] E. A. Martens, R. Kostadinov, C. C. Maley, and O. Hallatschek, Spatial structure increases the waiting time for cancer, New J. Phys. 13, 115014 (2011).

[22] N. Goldenfeld, Kinetics of a model for nucleation-controlled polymer crystal growth, J. Phys. A: Math. Gen. 17, 2807 (1984).

[23] C. Bennett, M. Büttiker, R. Landauer, and H. Thomas, Kinematics of the forced and overdamped sine-Gordon soliton gas, J. Stat. Phys. 24, 419 (1981).

[24] R. Pyke, Spacings, J. R. Stat. Soc. B 27, 395 (1965).

[25] I. Cvijović, A. N. N. Ba, and M. M. Desai, Experimental studies of evolutionary dynamics in microbes, Trends Genet. 34, 693 (2018).

[26] B. H. Good, M. J. McDonald, J. E. Barrick, R. E. Lenski, and M. M. Desai, The dynamics of molecular evolution over 60,000 generations, Nature (London) 551, 45 (2017).

[27] M. Baym, T. D. Lieberman, E. D. Kelsic, R. Chait, R. Gross, I. Yelin, and R. Kishony, Spatiotemporal microbial evolution on antibiotic landscapes, Science 353, 1147 (2016).

[28] A. Nourmohammad, J. Rambeau, T. Held, V. Kovacova, J. Berg, and M. Lässig, Adaptive evolution of gene expression in Drosophila, Cell Rep. 20, 1385 (2017).

[29] R. Hermsen, On the rapidity of antibiotic resistance evolution facilitated by a concentration gradient, Phys. Biol. 13, 065003 (2016).

[30] J. B. H. Martiny, B. J. Bohannan, J. H. Brown, R. K. Colwell, J. A. Fuhrman, J. L. Green, M. C. Horner-Devine, M. Kane, J. A. Krumins, C. R. Kuske et al., Microbial biogeography: putting microorganisms on the map, Nat. Rev. Microbiol. 4, 102 (2006).

[31] M. R. Bennett and J. Hasty, Microfluidic devices for measuring gene network dynamics in single cells, Nat. Rev. Genet. 10, 628 (2009).

[32] H. Mao, T. Yang, and P. S. Cremer, A microfluidic device with a linear temperature gradient for parallel and combinatorial measurements, J. Am. Chem. Soc. 124, 4432 (2002).

[33] O. Pringault, R. de Wit, and P. Caumette, A Benthic Gradient Chamber for culturing phototrophic sulfur bacteria on reconstituted sediments, FEMS Microbiol. Ecol. 20, 237 (1996).

[34] O. Pringault and F. Garcia-Pichel, Monitoring of oxygenic and anoxygenic photosynthesis in a unicyanobacterial biofilm, grown in benthic gradient chamber, FEMS Microbiol. Ecol. 33, 251 (2000).

[35] R. Hermsen, J. B. Deris, and T. Hwa, On the rapidity of antibiotic resistance evolution facilitated by a concentration gradient, Proc. Natl. Acad. Sci. USA 109, 10775 (2012).

[36] Q. Zhang, G. Lambert, D. Liao, H. Kim, K. Robin, C.-k. Tung, N. Pourmand, and R. H. Austin, Acceleration of emergence of bacterial antibiotic resistance in connected microenvironments, Science 333, 1764 (2011).

[37] C. M. Jessup, R. Kassen, S. E. Forde, B. Kerr, A. Buckling, P. B. Rainey, and B. J. Bohannan, Big questions, small worlds: microbial model systems in ecology, Trends Ecol. Evol. 19, 189 (2004).

[38] R. Kassen and P. B. Rainey, The ecology and genetics of microbial diversity, Annu. Rev. Microbiol. 58, 207 (2004).

[39] P. B. Rainey, A. Buckling, R. Kassen, and M. Travisano, The emergence and maintenance of diversity: insights from experimental bacterial populations, Trends Ecol. Evol. 15, 243 (2000).

[40] M. Lässig, V. Mustonen, and A. M. Walczak, Predicting evolution, Nat. Ecol. Evol. 1, 0077 (2017).

[41] K. S. Korolev, M. J. Müller, N. Karahan, A. W. Murray, O. Hallatschek, and D. R. Nelson, Selective sweeps in growing microbial colonies, Phys. Biol. 9, 026008 (2012). 